\title{
The Effect on Switching Lifetime of Chromium Adhesion Layers in Gold-Coated Electrical Contacts under Cold and Hot Switching Conditions
}

\author{
A. P. Lewis, M. P. Down, C. Chianrabutra, L. Jiang \\ and S. M. Spearing \\ Electro-mechanical Engineering, \\ University of Southampton \\ Southampton, UK, SO17 1EN \\ \{a.p.lewis, mpd2g12, cc12g10, 1.jiang, \\ s.m.spearing\}@soton.ac.uk
}

\author{
J. W. McBride \\ University of Southampton Malaysia Campus \\ No. 3 Persiaran Canselor 1, Kota Ilmu, EduCity@ Iskandar \\ 79200, Nusajaya, Johor, Malaysia \\ jwm@soton.ac.uk
}

\begin{abstract}
Gold is commonly used for electrical contacts due to its many desirable electrical and mechanical properties. Throughout the switch lifetime, the contacts are required to survive a large number of opening and closing cycles and therefore it is important to understand the failure mechanisms. Adhesion layers (e.g. chromium or titanium) can be deposited to increase the adhesion of the gold layer to the contact surface. In this work, the inclusion of a chromium adhesion layer shows an improvement of the switching lifetime of gold-coated electrical contacts under cold and hot switching conditions. These testing conditions further the understanding of the failure mechanisms (e.g. fine transfer, etc.). The mechanism of failure when no chromium adhesion layer was used is attributed to delamination of the gold layer from one contact to the other. This failure mechanism is different in the cases where a chromium adhesion layer is included. We present a model which was developed in line with experimental results. These describe the effect of load current on material transfer between gold contacts and the contact failure.
\end{abstract}

Keywords-Electrical contacts switching lifetime, adhesion layers, failure mechanisms, modelling failure.

\section{INTRODUCTION}

Due mainly to its good conductivity and chemical inertness gold is commonly used as a material for electrical contacts [1]. In micro-fabrication processes such as sputtering and evaporation, the deposition of gold electrodes requires adhesion layers (for example chromium and titanium) [2, 3]. It is known that the adhesion layers promote adhesion to various substrates, such as silicon or silicon dioxide. Typically the addition of chromium or titanium adhesion layers increases the adhesion energy by a factor of 10 [4]. Vianco et al. showed that the inclusion of a thin chromium layer improved the adhesion strength of gold to single crystal quartz to $\sim 48 \mathrm{MPa}$; in the absence of the chromium layer the gold did not adhere [5].

A downside of using pure gold for electrical contacts is that due to its high ductility and low hardness, the adhesion voltage

This work was supported by the Innovative Electronics Manufacturing Research Centre (IeMRC) and Engineering and Physical Sciences Research Council (EPSRC) under grant number: EP/H03014X/1. is lower than many other metals $[1,6]$. The tradeoff in terms of contact resistance and reliability of contact materials for high power RF-MEMS switches was discussed by Kwon et al. [7]. For example the use of iridium for the contact material enabled longer lifetimes at the cost of higher contact resistance. A tradeoff between contact resistance and reliability could be achieved by making one contact iridium and the other gold. The power handling capability of a gold-iridium contact pair was a $2.8 \mathrm{x}$ improvement over gold-gold [7]. Whilst increasing the contact material hardness increases the reliability, the effect on the surface roughness (and consequently the contact resistance) needs to be monitored [8]. Yang et al. investigated the use of nickel-gold alloys to achieve a balance between material hardness and contact reliability [8]. Mulloni et al. investigated a number of thin gold-based multilayers, detailing the resulting resistivity, surface roughness, hardness and surface composition following a variety of fabrication processes [1].

The effect of electrical current on the failure of gold contacts was investigated by $\mathrm{Kim}$ et al. [9]. As well as measuring the contact resistance, they measured the penetration depth into the surface of the gold contacts was measured using a nano-indenter; the nano-indenter also applied the contact force $(0.8 \mathrm{mN})$. With no current flowing through the contact, there was no noticeable change in penetration depth into the contact, even after repeated opening and closing of the contacts. When hot switching the contacts with currents up to $500 \mathrm{~mA}$, a change in the penetration depth was detected, following a number of switching cycles (depending on the current). The current caused Joule heating, leading to the melting of the gold contact [9]. The melted contact regions were deformed and pulled from the flat surface. This resulted in a decrease in contact area and increase in contact resistance and therefore failure of the contact surface [9]. At the point of failure, a change in the penetration depth was observable; therefore it was concluded that the penetration depth could be used to indicate contact failure. Larger currents caused 
increased softening and melting of the surface and therefore reduced the contact lifetime.

Even though adhesion layers are commonly used, their effect on the lifetime and failure mechanisms for switching contacts has received little or no attention in the reported literature. This work investigates some fundamental questions about the effect of adhesion layers for micro-fabricated contacts employed in switches. Primarily the use of chromium as an adhesion layer and its effect on the lifetime of electrical contacts will be discussed. We propose a failure mechanism, defined by the increasing adhesive force between the contacts overcoming the bond between film and substrate. The model of this mechanism describes the advantage to the switching lifetime brought about by the inclusion of an adhesion layer.

\section{BACKGROUND}

The failure of electrical contacts is complicated by the large number of potential failure mechanisms. Gilbert et al. recently identified three possible causes of failure for their gold contacts; these being: ductile separation, delamination and brittle separation [10]. The films were sputter coated with a 10 $\mathrm{nm}$ chromium adhesion layer followed by a $300 \mathrm{~nm}$ gold layer. Where the failure mechanism was due to ductile separation, the failure occurred within 10,000 to 70,000 switching cycles. The contact film delamination failure mode occurred on samples which failed between 200,000 and 500,000 cycles. For samples which switched for over $1,000,000$ cycles the failure was due to brittle separation. In our model we consider the increase in the adhesive force over a number of switching cycles. If there are defects in the layers this will reduce the critical adhesion force required for contact failure, resulting in premature failure. Due to the stochastic nature of defects, the range of reported values for contact lifetimes varies significantly. A discussion on the plastic, elastic and elastic-plastic deformation mechanisms of the contact material was given by Coutu et al.; their work related the deformation and contact force [11].

Both electrical and mechanical processes are involved in the switching cycles which can potentially be linked to failure mechanisms as detailed in the following sections.

\section{A. Electrical Considerations}

In the typical case, where a current is flowing between the two contacts, as the contacts separate the voltage across them increases rapidly to the voltage of the applied load. If this voltage is greater than the melting voltage of the contact material, as the contacts separate the final asperities in contact will melt [12]. The melting and boiling voltages for gold are $0.43 \mathrm{~V}$ and $0.88 \mathrm{~V}$ respectively [13]. The melted contact material can then be extruded to form a molten metal bridge. Since the voltage across the switch increases rapidly to the applied load voltage, the temperature of the molten metal bridge will increase. At the boiling voltage the material boils which breaks the bridge. Since the bridge does not necessarily break in the center, material can transfer between the contacts by this mechanism; this is referred to as the fine transfer mechanism [12].

\section{B. Mechanical Considerations}

Due to the repeated loading and unloading of the surface over a large number of cycles, it is expected that a series of mechanical effects can take place. Fatigue effects are expected to occur, with crack initiation and propagation, however with a low applied force and the negligible mechanical influence of the thin film the difference between the gold and chromiumgold contact pairs are distinguished only by a difference in values of shear yield stress and energy release rates.

It is observed that during closing and opening of the switch the conditions are suitable for the melting of material at the contact spots between the two rough surfaces [12]. However when the switch is closed, the potential across the switch tends toward zero, (though there will always be a constriction resistance), therefore the contact spots are believed to solidify causing the contacts to weld together [14]. The process is reversed during the opening, where the welded contact spots are stretched until the potential across the switch reaches the melting potential, at which point the contact bridge melts and breaks. We propose that the welds exert a peeling force between the contacts during opening, lifting the film from the substrate [15]. After a number of cycles it is believed that the surface asperities deform (flatten), effectively increasing the radius of the asperities [16]. The strength of the welds increases as the area increases, until the force from the welded contact spots can overcome the adhesion between the gold film and the substrate [16]. The influence of the presence of chromium is that the adhesive strength per unit area at the gold-silicon interface is greatly increased.

\section{PROPOSED FAILURE MODEL}

The contact area between asperities can be described readily by the JKR contact theory [17] in which the Hertzian contact theory is adjusted to account for the adhesive forces between the surfaces, which at the scale of asperity-asperity contact becomes a dominant property. The JKR theory gives the contact radius, $a$, to be:

$$
a_{J K R}^{3}=\frac{3 R}{4 E^{*}}\left(L+3 \pi \mu r+\sqrt{6 \pi r \mu L+3 \pi r \mu^{2}}\right)
$$

Where $L$ is the applied load, $\mu$ is the surface energy per unit area and $r$ is the relative radius of the asperities in contact such that:

$$
r=\frac{r_{1} r_{2}}{r_{1}+r_{2}}
$$

Where $r_{1}$ and $r_{2}$ are the radii of the respective asperities, and $E$ is the corrected Young's Modulus:

$$
E^{*}=\frac{1-v_{1}^{2}}{E_{1}}+\frac{1-v_{2}^{2}}{E_{2}}
$$

Where $v$ is Poisson's ratio, with the subscripts 1 and 2 identifying the material of each of the contacts materials.

JKR describes the adhesive contact between two spheres, however if we consider a current passing through the spheres a variable value for the hardness is required. This variation should account for the softening of the material with current. A larger current passing through the contact results in an increase in material softness and consequently a larger contact area and resultant weld.

The welded force between two asperities is calculated by considering the two asperities as a cylindrical body (or wire) beings stretched, whose original length, $l$, is the sum of the 
heights of the two asperities. The force to extend these welded asperities by displacement, $\Delta x$, is given by:

$$
F=\frac{E^{*} A \Delta x}{l}
$$

Where $A$ is the contact area $\left(A=2 \pi a^{2}\right)$, we can see that if the asperities deform plastically during repeated loading the force increases as a function of $a_{J K R}^{2}$. The total weld strength is expected to increase until strong enough to overcome the adhesive, interfacial bonds between the conductive film and the silicon substrate. The weld melts and breaks when the voltage through the 'wire' is the melting voltage, $V$, of the switch material:

$$
V=I R_{e}=\frac{I \rho}{2 A}
$$

Here, $\rho$ is the material resistivity, $I$ is the current and $R_{e}$ is the electrical resistance of the contacts defined by the contact constrictive resistance. If we assume a current of $50 \mathrm{~mA}$, and given the melting voltage of gold is $0.43 \mathrm{~V}$ [13] we get a critical radius across the 'wire' of $2.099 \mu \mathrm{m}$. When the area reaches this value due to separation the bridge melts and breaks, and the lifting force ceases. As a result, neglecting the non-linearities and time-dependent effects, the maximum force between the contact surfaces before melting is given by:

$$
F_{\mathrm{m}}=\frac{E^{*} I \rho \Delta x}{2 V_{m}}
$$

If we consider the asperities to have elastic-plastic characteristics during loading, the critical vertical displacement during the closing stage of the process is given by [11]:

$$
x_{c}=r\left(\frac{H \pi}{2 E^{*}}\right)^{2}
$$

At which a vertical deformation is observed:

$$
x_{\mathrm{d}}=\frac{1}{3}\left(\frac{H \pi r^{2} x_{c}}{H \pi r^{2}-L_{c}}\right)
$$

Where $H$ denotes hardness and $L_{c}$ is the load at which deformation occurs:

$$
L_{c}=\left[1-\left(\frac{x_{c}}{3 x}\right)\right] H A
$$

The changes to each asperity are expected to be small however the number of asperities being expected to be in the hundreds at the point of contact with a pressure distribution according to the spherical contact the growth of the contact areas increases gradually, until the maximum lift-off force is large enough to overcome the shear yield stress of the laminated surface.

According to $\mathrm{L}$. Wu et al. [16] after the critical load has been applied and the critical deformation has been surpassed the spherical asperity returns to a shape with a radius of:

$$
r_{r}=r\left[1+1.275\left(\frac{H}{E}\right)^{0.216}\left(\frac{x}{x_{c}}-1\right)\right]
$$

If the asperity experiences a loading and unloading over $N$ cycles, to the maximum reach of the system, $x$, then:

$$
r_{r}=r_{0}[1+N k]
$$

Where $N$ is the number of cycles and:

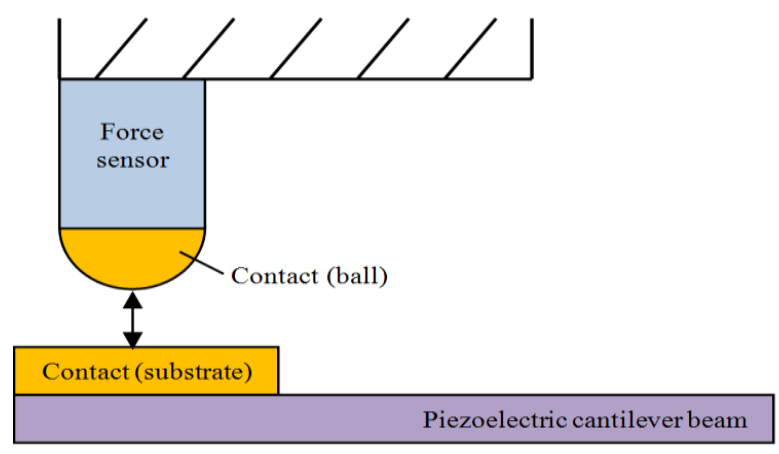

Fig. 1. Schematic of experimental rig.

$$
k=1.275\left(\frac{H}{E}\right)^{0.216}\left(\frac{x}{x_{c}}-1\right)
$$

The evolution of $r$ as a result of elastic-plastic deformation of the asperities, under cyclic loading, increases the contact area and consequently the adhesive forces and welding strength between the two contacts.

By analysis we can see that if the JKR theory accounts for the radius of the contact asperities as a function of the number of cycles, the contact area, and hence force, are proportional to $N^{2 / 3}$.

This can be observed more readily in the case of gold sputtered onto a silicon substrate where an oxide layer $\left(\mathrm{SiO}_{2}\right)$ is formed, due to the poor work of adhesion at the interface [18]. A low number of cycles create an area large enough for a weld of strength greater than the bond between the noble metal and the oxide metal layer. The presence of chromium however allows for different oxides to form at the interface. The valence states allow more options for oxide layers to be formed at the interface; as a result at the interface oxide-oxide interatomic bonds form, metal-oxide bonds, and metal-metal bonds at the gold-chromium interface [18]. The strength of these bonds significantly is greater than the weak metal-oxide bonds between the noble metal (gold) and the oxide layer $\left(\mathrm{SiO}_{2}\right)$.

\section{EXPERIMENTAL SETUP}

The experimental setup consisted of two contacts; the first being a gold-coated stainless steel ball and the second being a gold-coated silicon oxide/silicon substrate (the silicon substrate has a $1 \mu \mathrm{m}$ thick thermal oxide layer). The balls and substrates were sputter coated with $500 \mathrm{~nm}$ of gold (sputtering machine: Kert J. Lesker sputterer). For the samples where a chromium adhesion layer was used, the thickness of this layer was $20 \mathrm{~nm}$. The gold-coated ball is attached to a low-level force sensor (Kistler Type: 9207), which is fed directly into a charge amplifier. The substrate was adhered onto a piezoelectric cantilever beam as shown in Fig. 1. The actuation of the cantilever beam brings the substrate into contact with the goldcoated ball. In this work the contact force was kept constant at $1 \mathrm{mN}$ in line with previous work and values typical for MEMS relays [19-22]. The load voltage was $4 \mathrm{~V}$; this was switched using a square wave with a frequency of $20 \mathrm{~Hz}$, which gave a contact duration of $25 \mathrm{~ms}$ per cycle. The load current was varied between $0-50 \mathrm{~mA}$. During the lifetime experiments, the contact resistance was measured periodically using a four-wire measurement method using a micro-ohmmeter (Keithley 580). SEM images were obtained for the all the contact pairs tested. 

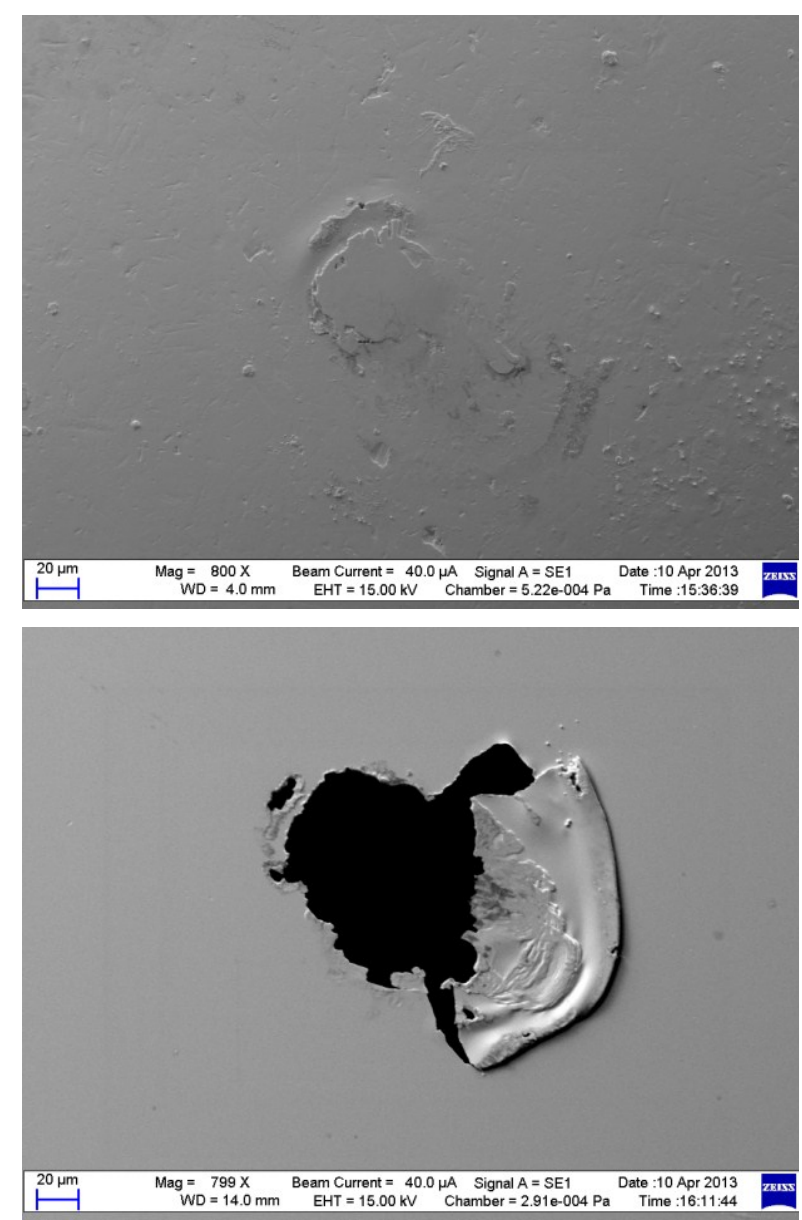

Fig. 2 SEM image of Au-coated contacts (top: ball, bottom: substrate) from experiment where $50 \mathrm{~mA}$ was switched. After $\sim 600,000$ cycles the contact failed.

\section{RESULTS}

Switching experiments were run for the following contact pairs:

- Au-coated ball to Au-substrate,

- CrAu-coated ball to Au-coated substrate,

- CrAu-coated ball to CrAu coated substrate.

For each contact pair the contact resistance was recorded periodically over the switching lifetime of the contacts. For the cold-switching tests, no current was passed through the contact during switching, whereas for the hot-switching tests a load current of $50 \mathrm{~mA}$ was used. To evaluate the effect of the current level on the contact lifetime, the $\mathrm{CrAu}$-coated ball to $\mathrm{Au}$-coated substrate was also tested at $10 \mathrm{~mA}$ and $30 \mathrm{~mA}$.

\section{A. Au-Coated Ball to Au-Coated Substrate}

After switching with a load current of $50 \mathrm{~mA}$ for $\sim 600,000$ cycles the contact resistance of the $\mathrm{Au}-\mathrm{Au}$ contact pair increased significantly indicating contact failure. An SEM image of the resulting contact surfaces can be seen in Fig. 2. The failure appears to be caused by the delamination of the gold from the substrate to the ball. To determine whether the cause of the delamination was primarily due to mechanical or electrical causes, the $\mathrm{Au}-\mathrm{Au}$ contact pair was run for the same
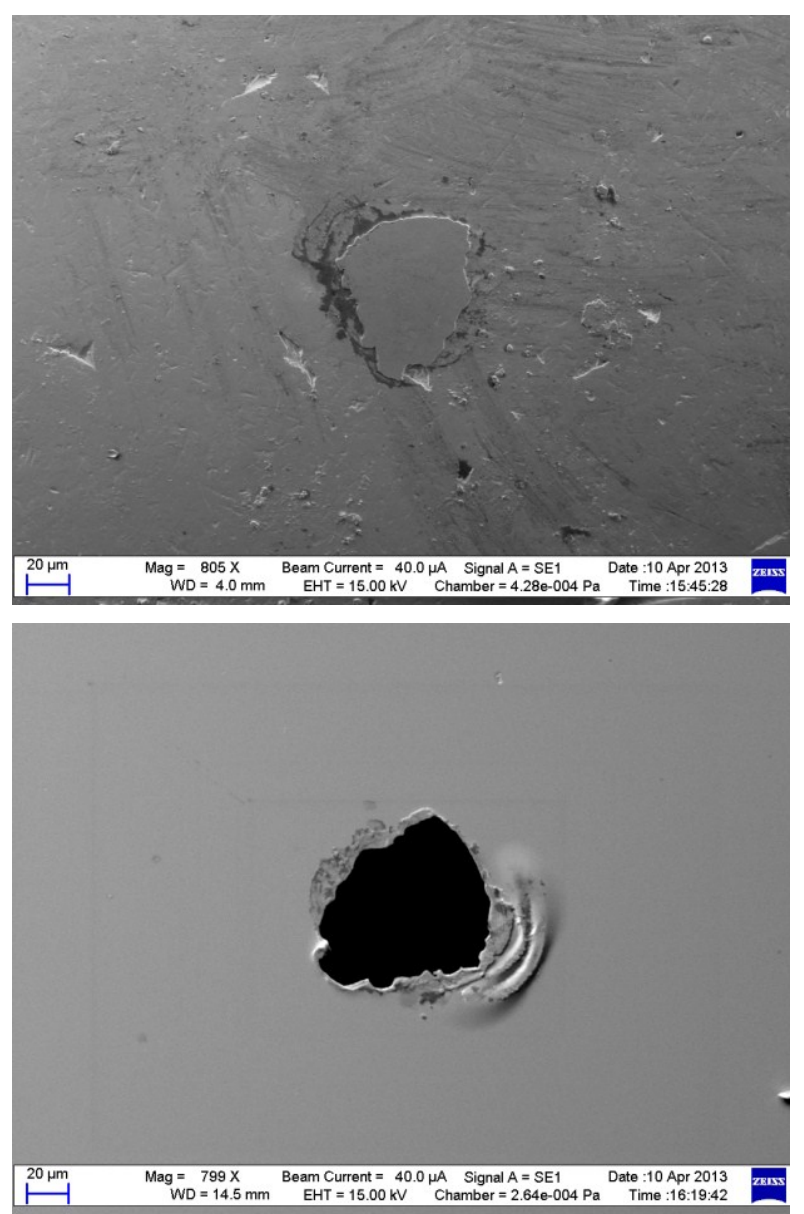

Fig. 3. SEM image of Au-coated contacts (top: ball, bottom: substrate) from experiment where there was no current flow during switching; switched for $\sim 600,000$ cycles.

number of cycles but with no load current. SEM images of both contact surfaces, after 600,000 cycles of cold switching, can be seen in Fig. 3. These show that a significant area of the contact has delaminated from the substrate to the ball. This suggests that the delamination of the contact is caused by a mechanical mechanism and is not purely electrical.

\section{B. CrAu-Coated Ball to Au-Coated Substrate}

The CrAu-coated ball and Au-coated substrate contact pair was tested with a range of load currents. The results from these experiments can be seen in the graph in Fig. 4. It can be seen

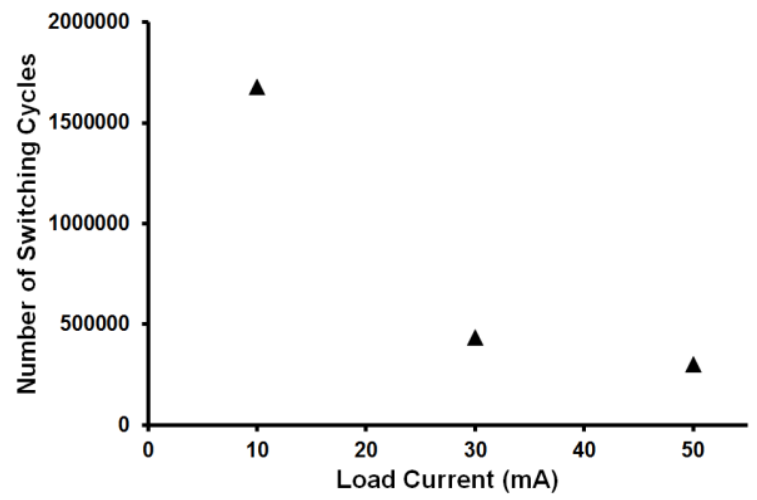

Fig. 4. Graph showing effect of load current on the contact pair lifetime where the contact pair is a CrAu ball to Au substrate. 
that the lifetime of the contact pair is related to the load current. With a low level of load current $(10 \mathrm{~mA})$, the contact pair survived for 1.67 million cycles. Failure occurred within 300,000 cycles when a high load current was used. Using an SEM, the surfaces of all of the failed contacts were analyzed. Similarly to the $\mathrm{Au}-\mathrm{Au}$ contact pair, the failure mechanism was attributed primarily to the delamination of the gold from the substrate to the ball.

The experimental results can be combined with the model, proposed in Section III, to calculate a critical adhesive force which needs to be overcome to result in contact failure. The experimental data which showed that the gold-coated substrate (no chromium) failed after 300,000 cycles (where $I=50 \mathrm{~mA}$ ). If the average radius of each contact asperity is assumed to be $0.5 \mu \mathrm{m}$, the average load on all asperities is $1 \mathrm{mN}$ and that the number of asperities in contact is 100 , the model gives a critical adhesion force of $\sim 0.89 \mathrm{mN}$. The assumptions are based on observations from unpublished work. It was also assumed that the asperities on the contact and ball are the same height and radius, and that the natural length of the 'wire' element is the sum of these asperity heights.

\section{CrAu-Coated Ball to CrAu-Coated Substrate}

The inclusion of a chromium adhesion layer on the substrate significantly improved the switching lifetime of the
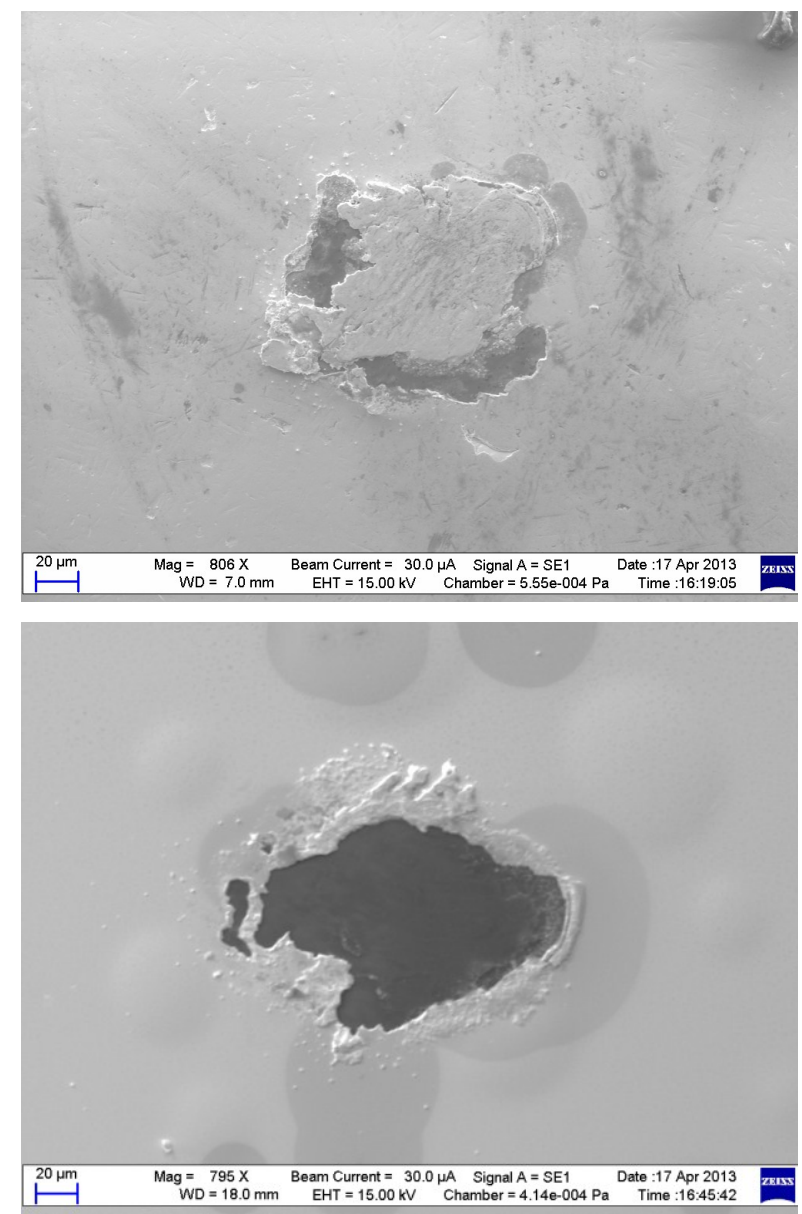

Fig. 5. SEM image of CrAu contacts (top: ball, bottom: substrate) from experiment where $50 \mathrm{~mA}$ was switched. After 10,300,000 cycles the contact failed.

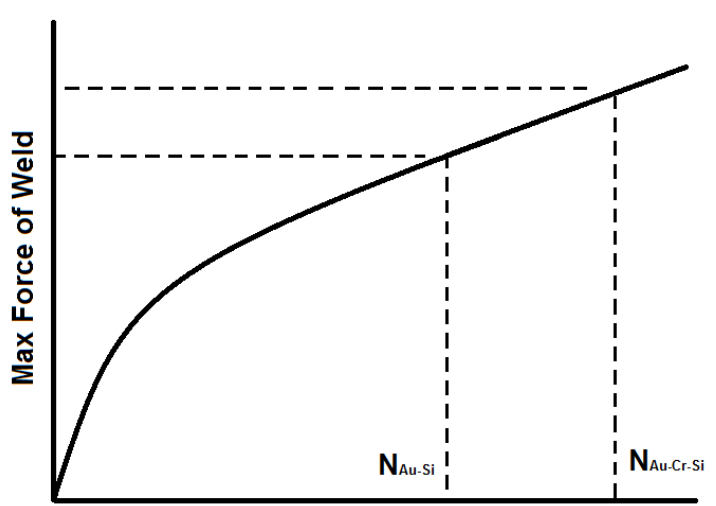

Number of Cycles

Fig. 6. Graph showing the variation in adhesive force versus the number of switching cycles

contact. With a load current of $50 \mathrm{~mA}$, the contact pair survived 10,300,000 switching cycles before failure; an image of the resulting surfaces can be seen in Fig. 5. It is clear that delamination of the gold film has occurred; however the SEM image of the substrate reveals two other interesting effects not observed with the Au-coated substrates (without an adhesion layer). The first being the presence of numerous specks of material around the edge of the contact area, these may be caused by the fine transfer mechanism. The other effect is that the film appears to blisters outside of the contact area. Using the same approach and assumptions as discussed in Section B of $\mathrm{V}$, it is possible to calculate the critical adhesion force for the chromium-gold contact. The contact lifetime of 10,300,000 cycles results in a critical adhesion force of $\sim 3.49 \mathrm{mN}$. The calculated values for the force are encouraging since the relationship between the force and number of cycles is not a linear relationship but a $N^{2 / 3}$ relationship. An illustration of how the weld force increases with the number of cycles can be seen in Fig. 6.

\section{DISCUSSION}

In the experiments with the gold-coated substrate, where no chromium adhesion layer was employed, the failure occurred significantly earlier than for the case where a chromium adhesion layer was used. It is well established that the adhesion of gold to silicon and silicon dioxide is poor, and so the use of adhesion layers such as chromium is common in metal deposition processes. An understanding of the effect of adhesion layers for electrical contacts in important, especially for determining the consequences on the switch lifetime. The relationship between the number of cycles and the asperity radius is given in (11). Our model describes how the contact area and consequently the welding strength between two contacts increase with the number of cycles. The inclusion of an adhesion layer results in the requirement for a greater welding strength in order to overcome the improved adhesion. The improvement in the adhesion strength when using an adhesion layer results in an increase in the critical welding strength of the contact pair. Therefore a greater number of switching cycles are required to reach this threshold, after which delamination can occur, resulting in the failure of the switch. Using this relationship it is possible to describe, by 
calculating a critical welding force, how the inclusion of an adhesion layer improves the switching lifetime of contacts.

The effect of the load current on the switching lifetime was measured for the gold-coated substrate. At higher current levels, the lifetime decreases significantly. This shows that the failure mechanism is not purely mechanical but due to a combination and interaction between of the electrical and mechanical processes. A number of experiments using goldcoated substrates with and without the inclusion of a chromium adhesion layer were tested under hot- and cold-switching conditions. Whilst delamination of the substrate contact material occurred under both conditions, failure occurred within fewer switching cycles at higher currents. Larger currents can lead to an increase in the temperature of the contact surfaces. Higher temperatures lead to a reduction in the hardness of the material. As can be seen in the model, the hardness is an important factor in the failure mechanism. Further to this, with a larger flow of current, the contacts are subjected to the potential of greater localized melting/boiling damage.

From our experiments it is clear that the inclusion of a chromium adhesion layer altered the failure mechanism. In addition to delamination, SEM images of the surfaces show the transfer of small areas of the substrate contact material, as well as a blistering of the thin film. Further to this the adhesion layer reduces the lateral displacement of the thin film. The SEM images suggested that the failure is primarily due to the delamination of the gold film. For substrates with no adhesion layer, this delamination occurred with and without current flow. There was no indication of fine transfer, although this evidence could be hidden under the delaminated film. For the chromium-gold contact however, there are a number of small areas of material transferred from the substrate to the ball which are indicative of a fine transfer process. A delamination process was also clearly present and suspected to be the dominant cause of failure.

Work by Kim et al. investigated the penetration depth of a titanium-gold contact when switching with a force of $0.8 \mathrm{mN}$ for a few hundred cycles; they stated that there was no remarkable change when there was no current flowing through the contacts [9]. They reported cycles to failure for their contacts over a range of currents, from $10 \mathrm{~mA}$ up to $500 \mathrm{~mA}$. At $10 \mathrm{~mA}$ their contact failed after 500 cycles which is significantly less than any of the contacts tested in our experiments. The primary difference being the contact time; in our work the contacts are switched at $20 \mathrm{~Hz}$, therefore the contact time is $\sim 25 \mathrm{~ms}$. This is significantly less than the $5-10 \mathrm{~s}$ used by Kim et al. This suggests that the contact time is an important factor which needs consideration in order to evaluate the performance of an electrical contact.

\section{CONCLUSIONS}

Fine transfer models which have been used to describe failure of electrical contacts are not sufficient to describe the delamination processes which contributed to the failure of the contacts described in this work. The use of a chromium adhesion layer was shown to improve the lifetime performance of electrical contacts. Whilst the failure mechanism was primarily due to delamination, independent of the presence of a chromium adhesion layer, SEM images of the failed contact surfaces revealed that the presence of a chromium adhesion layer resulted in a number of other processes which affected the surface. These included blistering of the thin film and the transfer of small volumes of material.

Using a relationship between the switch current and the contact area of the molten metal bridge we combined the electrical and mechanical elements of the model to describe the failure. The model accounts for the improvement in adhesion provided by the adhesion layer by adjusting the shear yield stress of the contact. Using the model presented it is possible to calculate a critical welding force which is dependent on the number of switching cycles. The adhesive welding force of the contact increases with the number of switching cycles. Once the critical welding force has been exceeded by the adhesive welding of the switching contacts, delamination can occur. Combining the model and experimental data a critical adhesion force was calculated for the contacts pairs, both with and without, the inclusion of a chromium adhesion layer. It was shown that the inclusion of a chromium adhesion layer increased this threshold force from $0.89 \mathrm{mN}$ to $3.49 \mathrm{mN}$.

\section{REFERENCES}

[1] V. Mulloni, J. Iannacci, R. Bartali, V. Micheli, S. Colpo, N. Laidani, and B. Margesin, "Gold-based thin multilayers for ohmic contacts in RFMEMS switches," pp. 80661D-80661D, 2011.

[2] C. W. Tan and J. Miao, "Optimization of sputtered $\mathrm{Cr} / \mathrm{Au}$ thin film for diaphragm-based MEMS applications," Thin Solid Films, vol. 517, pp. 4921-4925, 2009.

[3] M. Schneider, H. Möhwald, and S. Akari, "Quantitative measurement of chromium's ability to promote adhesion," The Journal of Adhesion, vol. 79, pp. 597-607, 2003/06/01 2003.

[4] A. G. Evans, J. W. Hutchinson, and Y. Wei, "Interface adhesion: effects of plasticity and segregation," Acta Materialia, vol. 47, pp. 4093-4113, 1999.

[5] P. T. Vianco, W. R. Conley, and J. K. G. Panitz, Resistivity, adhesive strength, and residual stress measurements of thin film metallizations on single crystal quartz, 1990.

[6] X. Lafontan, F. Pressecq, G. Perez, C. Dufaza, and J. M. Karam, "Physical and reliability issues in MEMS microrelays with gold contacts," pp. 11-21, 2001

[7] H. Kwon, D.-J. Choi, J.-H. Park, L. Hee-Chul, Y.-H. Park, Y.-D. Kim, H.-J. Nam, Y.-C. Joo, and J.-U. Bu, "Contact materials and reliability for high power RF-MEMS switches," in IEEE 20th International Conference on Micro Electro Mechanical Systems, 2007. MEMS., 2007, pp. 231-234.

[8] Y. Zhenyin, D. J. Lichtenwalner, A. S. Morris, J. Krim, and A. I. Kingon, "Comparison of Au and Au-Ni Alloys as Contact Materials for MEMS Switches," Microelectromechanical Systems, Journal of, vol. 18, pp. 287-295, 2009.

[9] D. S. Kim, S.-D. Ko, S. J. Lee, B.-K. Lee, J.-B. Yoon, and D. K. Kim, "Investigation on the Electrical Contact Behaviors of RF MEMS switches using nano-indenter," Review on Advanced Material Science, vol. 28, pp. 17-20, 2011.

[10] K. W. Gilbert, S. Mall, and K. D. Leedy, "Characterization of GoldGold Microcontact Behavior Using a Nanoindenter Based Setup," 
Journal of Adhesion Science and Technology, vol. 24, pp. 2597-2615, 2010/01/01 2010.

[11] R. A. Coutu Jnr and P. E. Kladitis, "Contact Force Models, including Electric Contact Deformation, for Electrostatically Actuated, CantileverStyle, RF MEMS Switches," in NSTI Nanotechnology Conference and Trade Show, 2004, pp. 219-222.

[12] F. Llewellyn-Jones, The Physics of Electrical Contacts: Clarendon Press, 1957.

[13] J. W. McBride, "The Wear Processes of Gold Coated Multi-walled Carbon Nanotube Surfaces used as Electrical Contacts for Micro-electromechanical Switching," Nanoscience and Nanotechnology Letters, vol. 2, pp. 357-361, 2010.

[14] B. Bhushan, "Adhesion and stiction: Mechanisms, measurement techniques, and methods for reduction," Journal of Vacuum Science \& Technology B: Microelectronics and Nanometer Structures, vol. 21, pp. 2262-2296, 2003.

[15] A. G. Evans and J. W. Hutchinson, "On the mechanics of delamination and spalling in compressed films," International Journal of Solids and Structures, vol. 20, pp. 455-466, 1984.

[16] L. Wu, J. C. Golinval, and L. Noels, "A micro-model for elasto-plastic adhesive-contact in micro-switches: Application to cyclic loading," Tribology International, vol. 57, pp. 137-146, 2013.

[17] K. L. Johnson, K. Kendall, and A. D. Roberts, "Surface Energy and the Contact of Elastic Solids," Proceedings of the Royal Society of London.
A. Mathematical and Physical Sciences, vol. 324, pp. 301-313, September 8, 19711971.

[18] V. E. Henrich and P. A. Cox, The Surface Science of Metal Oxides: Cambridge University Press, 1996.

[19] D. Hyman and M. Mehregany, "Contact physics of gold microcontacts for MEMS switches," Components and Packaging Technologies, IEEE Transactions on, vol. 22, pp. 357-364, 1999.

[20] M. Vincent, S. W. Rowe, C. Poulain, D. Mariolle, L. Chiesi, F. Houze, and J. Delamare, "Field emission and material transfer in microswitches electrical contacts," Applied Physics Letters, vol. 97, pp. 263503-3, 2010.

[21] J. W. McBride, L. Jiang, and C. Chianrabutra, "Fine Transfer in Electriacl Switching Contacts Using Gold Coated Carbon-Nanotubes," in Joint Conference of 26th International Conference on Electrical Contacts and 4th International Conference on Reliability of Electric Products and Electric Contacts, Beijing, 2012.

[22] J. W. McBride, S. M. Spearing, L. Jiang, and C. Chianrabutra, "Gold Coated Carbon-nanotube Surfaces as Low Force Electrical Contacts for MEMS Devices: Part II, Fine Transfer Mechanisms," presented at the 57th IEEE Holm Conference on Electrical Contacts, Minniapolis, MN, 2011. 